\title{
Introduction : La part « d'immatériel » dans les objets de culture dite " matérielle »
}

Isabelle Leblic

\section{OpenEdition}

1 Journals

Édition électronique

URL : http://journals.openedition.org/jso/6839

DOI : $10.4000 /$ jso.6839

ISSN : 1760-7256

\section{Éditeur}

Société des océanistes

\section{Édition imprimée}

Date de publication : 15 octobre 2013

Pagination : 5-15

ISBN : 978-2-85430-035-2

ISSN : 0300-953x

Référence électronique

Isabelle Leblic, «Introduction : La part "d'immatériel » dans les objets de culture dite " matérielle » », Journal de la Société des Océanistes [En ligne], 136-137 | 2013, mis en ligne le, consulté le 22

septembre 2020. URL : http://journals.openedition.org/jso/6839; DOI : https://doi.org/10.4000/jso. 6839 


\section{Introduction : La part d' "immatériel » dans les objets de culture dite " matérielle »}

par

Isabelle LEBLIC ${ }^{*}$

À l'automne 2013, Emmanuel Kasarhérou et Roger Boulay, sociétaires de la société des Océanistes, présentent au musée du quai Branly une exposition de plus de 300 objets et documents de la culture matérielle kanak, issus des collections des musées d'Europe (Autriche, Suisse, France, Allemagne et Italie) et de Nouvelle-Calédonie, en insistant sur la relecture que l'on peut faire aujourd'hui d'un patrimoine matériel conservé. Organisée autour de deux principes: "les Kanak par eux-mêmes " et "Kanak et Européens échangeant leurs regards", Kanak, l'art est une parole (au MQB du 15 octobre 2013 au 26 janvier 2014) sera, du fait de la présentation de pièces spectaculaires et inédites telles que sculptures fâ̂tières et chambranles sculptés de cases traditionnelles, haches ostensoirs de jade mises face à des haches européennes, statuettes et objets rituels divers, etc. (voir le cahier photos reproduisant certains des objets prêtés par le musée de Nouvelle-Calédonie), la plus importante exposition sur la Nouvelle-Calédonie kanak depuis plus de vingt ans. Pour l'accompagner, la rédaction du Journal de la Société des Océanistes a choisi de préparer un volume susceptible d'intéresser tant les visiteurs de cette exposition que les lecteurs habituels du journal. Intitulé La part "d'immatériel " dans les objets de culture dite "matérielle ", ce dossier a voulu mettre un accent particulier sur la culture matérielle et immatérielle kanak, en l'ouvrant à d'autres cultures océaniennes afin d'enrichir, par la comparaison, les réflexions sur cette thématique. Dans notre appel à contributions, nous avions privilégié plusieurs thèmes qui nous semblaient d'importance. Nous les reprendrons ici pour cerner mieux notre propos, en regrettant que certains n'aient pas donné lieu à des propositions d'articles.

Une première question est bien sûr de déterminer ce que cette distinction entre "matériel " et " immatériel » a de recevable ou de compatible du point de vue des civilisations dites "traditionnelles» ou autochtones du Pacifique ou, au contraire, si elle n'est pas entièrement d'importation, et principalement à usage colonial - puis néocolonial aujourd'hui. La contribution de Pierre Lemonnier revient d'emblée sur cette distinction importante dans son article intitulé "De l'immatériel dans le matériel... et réciproquement! Techniques et communication non verbale ». Plusieurs choses viennent à l'esprit à ce propos, et notamment le lien entre le domaine social et le domaine technique :

«l'étude de la vie matérielle des hommes n'a de signification que si la technologie possède une lucide conscience sociologique des faits étudiés. [...] [Ses] matériaux ne prendront vie que s'ils sont élaborés en fonction des rapports économiques, esthétiques et sociaux qui les unissent, non uniquement à l'artisan, mais à la collectivité plus ou moins large à laquelle il appartient. »(Leroi-Gourhan, 1952 : 515-516)

Georges Guille-Escuret, en introduction d'un volume sur l'«efficacité technique, l'efficacité sociale " (2003) posait la question de savoir si le technique est dans le social ou face à lui. Les connaissances techniques - à la fois discours des acteurs et savoirs implicites auxquels l'observa- 
teur, l'ethnologue, ne peut avoir accès directement -, comme les savoir-faire sont deux choses indispensables non seulement pour la compréhension de tout processus technique mais également pour sa réalisation. Il y a trente ans, on considérait que ces connaissances techniques étaient la part de la technique qui "met en lumière des interrelations entre le "technique" et le "social" "(Chamoux, 1983 : 99). Depuis, dans leurs travaux récents, Coupaye $(2009,2012)$, Damon (2008) et Lemonnier (2012) ont établi que les manières de faire en elles-mêmes, qui ne se limitent pas seulement aux connaissances ou aux représentations des éléments techniques, sont liées à des manières de vivre, au "social ", en insistant sur la dimension de communication non verbale contenue dans les objets et actes techniques, qui permettent par exemple de dire sans mots des aspects essentiels d'une façon de vivre ensemble ${ }^{1}$. Comme le souligne Pierre Lemonnier dans l'introduction de son dernier livre :

" This book shows why and how a few artefacts lead the actors to aggregate various aspects of their social life and thus enable them to seize nonpropositional clues underlying key social relations. [...] In other words, by studying such ordinary things as New Guinea garden fences, traps, and magical bundles, as well as European toy cars, I try to understand why material objects and actions lend themselves particularly well to blending thoughts, which in turn allows the actors to mentally grasp cardinal social relations and values underlying their daily life.» (2012:14)

Ainsi, cette question de l'immatériel dans le matériel pose d'une autre façon la question du lien entre la technique et la société. Comme le rappelle Georges Guille-Escuret (2003), Robert Cresswell (1994 : 275) a établi « la nature cyclique des relations entre le technique et le social ", " une homologie entre systèmes techniques et sociaux" après que Leroi-Gourhan (1952 : 515) eut bien précisé que " [la] fin n’est pas dans l'étude des techniques humaines, mais dans l'étude de l'homme qui pense et agit techniquement ». Depuis, Pierre Lemonnier entre autres (2011a, b et ci-après : «Effet, reflet, correspondance ${ }^{2}$, compatibilité...: de l'art de replacer un système technique dans la configuration culturelle qui l'englobe», pp. 17-19), parlent de correspondances. La technologie culturelle ${ }^{3}$ que j'ai pratiquée pendant longtemps dans mes recherches en anthropologie maritime kanak comme sur les poteries kanak m'ont permis de montrer certaines de ces homologies entre systèmes techniques et systèmes sociaux, notamment en constatant que certains aspects de l'organisation sociale étaient fondés par les spécialisations techniques. Autrement dit, les rôles et les statuts des clans pêcheurs kanak à l'intérieur des entités sociopolitiques constituant les " pays ", qui sont insérés dans une hiérarchie interne entre clans anciens (autochtones) et clans de "serviteurs", sont directement liés à la détention de savoirs et savoir-faire techniques et rituels. Car, au niveau des représentations de la nature et de la surnature kanak, l'efficacité technique est en grande partie liée aux rituels propitiatoires et à l'intervention bienveillante des ancêtres. Comme je l'ai déjà écrit :

"Répondre du titre de clan de pêcheurs suppose être reconnu détenteur des connaissances et des objets magico-religieux (pratiques rituelles et propitiatoires) nécessaires à la capture des animaux marins. La pratique technique est ainsi subordonnée à ce pouvoir magique. Pour la pêche comme pour l'agriculture (ou toute autre activité), on peut considérer deux sortes de magie. L'une, générale et très puissante, dont l'efficacité, non limitée dans l'espace et dans le temps, agit de façon positive (pour la réussite d'une activité) ou négative (pour la faire échouer) sur l'ensemble d'un "pays" (l'île des Pins par exemple); elle est détenue par un membre des clans dits anciens (originaires, "maitres du terrain ") et le plus souvent, semble-til, aussi "gardiens" de la chefferie. L'autre, purement "familiale", qui n'agit que localement et temporairement pour favoriser la réussite de telle pêche ou de telle culture... Ainsi, à l'île des Pins, il existe deux catégories de clans de pêcheurs : les anciens et les "serviteurs". Les clans les plus anciens de l'île détiennent les grandes pratiques magiques pour la pêche et ont le statut élevé de premiers occupants du pays, de fondateurs de la "chefferie". Par conséquent, s'ils participent aux pêches "collectives", ils sont bien distingués des clans "serviteurs" qui pratiquent la pêche à la demande expresse du "grand chef" pour préparer les échanges cérémoniels ou répondre à un don d'ignames par un contre-don équivalent de poissons. Ces clans "serviteurs" sont soumis à l'autorité rituelle des clans de pêcheurs dits anciens. » (Leblic, 2008 : 229)

Le rapport "cyclique» des techniques avec la structure sociale se fait ici à plusieurs niveaux :

- D'une part, le lien entre "mythes et techniques » - «l'origine des manières de faire »(Jamard, 2004); nous avons montré comment

1. Je remercie Pierre Lemonnier d'avoir attiré mon attention sur cette question.

2. "On est passé des correspondances de domaines de la vie sociale supposées par l'anthropologue à un intérêt pour leur mise en correspondance par les acteurs. " (Lemonnier, ci-après : 20).

3. «La technologie culturelle n'est assurément qu’un point de vue [...] et ce point de vue n’est pas destiné à construire seulement un savoir, la "technique", mais un savoir sur les aspects les plus divers d'une culture ou d'une organisation sociale. [...] se pencher sur les dimensions les plus matérielles des objets, comprendre leur fabrication ou leur fonctionnement permet surtout de mettre au jour des pans entiers de systèmes de pensée ou de logiques sociales, qu'on ne saurait repérer et comprendre autrement. " (Lemonnier, 2004 : 30). Et, avec André-Georges Haudricourt (1987), nous rappellerons que la " technologie [est] science humaine". 
les techniques avaient une origine surnaturelle et que les humains se les appropriaient par vol, ruse ou don, ce que de nombreux mythes étiologiques $^{4}$ et/ou d'origine racontent (Leblic, 1988, 2008 ; voir aussi Juillerat, 2004).

- D'autre part, par la synergie que la répartition des clans dans diverses fonctions techniques et/ou sociales ${ }^{5}$ exerce :

"Ces spécialités techniques sont donc identificatoires et sont plus particulièrement mises en œuvre dans le cadre des activités cérémonielles. À ce titre, on peut dire qu'elles relèvent plus de l'idéologie que de la pratique. En effet, n'importe qui peut pềcher, sous réserve qu'il ne capture pas les espèces réservées aux échanges cérémoniels et qu'il respecte les interdits. La pêche n'est une véritable spécialité que dans ce cadre cérémoniel, ce qui contribue à expliquer les différents statuts présentés ci-dessus : pêcheur "ancien", pêcheur “étranger intégré comme chef”, etc. » (2008 : 230)

Dans le même esprit, les potiers - ou plutôt les potières car ce sont souvent des femmes qui étaient en charge de cette fabrication (Leblic, 1999) - sont aussi partie prenante de cette hiérarchie sociale où les fabricants de poterie sont liés à l'autochtonie; ainsi, les poteries sont des objets dotés d'un statut particulier, faisant partie des "richesses" des sociétés kanak, c'est-àdire des objets insérés dans les échanges entre groupes, marqueurs aussi des relations d'alliance et de hiérarchie. Toutes ces pratiques techniques sont donc subordonnées à la possession conjointe d'un savoir et d'objets magiques, avec la prise en compte des agents surnaturels que sont les diverses catégories d'esprits et d'ancêtres qui sont indispensables pour la compréhension des chaînes opératoires, que l'on peut définir comme :
" un instrument idéal pour révéler les connexions entre matérialité et idéalité. » (Coupaye, 2009 : 50)

\section{Comme l'a écrit Ludovic Coupaye :}

"L'ensemble des chaînes opératoires impliquées dans la culture des grandes ignames mobilise un ensemble hétérogène de gestes, d'actions sur la matière et le corps, de substances, d'acteurs, de lieux et de temporalités [...] qui ont tous une raison d'être spécifique et sont mobilisés pour atteindre un résultat efficace. L'objet fini est donc un condensé de relations sociales, matérielles, symboliques réalisées au cours de la chaîne opératoire et concrétisées sous la forme d'un seul artefact $[. .$.$] qui doit impérativement être donné$ à voir. » $(2009: 20)$

\section{Et, comme le souligne Jean-Pierre Warnier :}

"La technologie, comme savoir sur les techniques, s'applique non seulement aux matières et artefacts, mais également aux sujets et à leurs corps. » (2009 : 149)

Ludovic Coupaye et Laurence Douny précisent aussi que :

« [on trouve] dans l'étude des systèmes techniques et dans l'outil méthodologique offert par la chaîne opératoire, un appareil empirique adéquat pour analyser, dans le détail, la dimension culturelle (donc, relative), sociale (donc potentiellement construite), évolutive (donc historique) et fonctionnelle (ethnologique) de la vie matérielle. » $(2009: 25)$

Ainsi, cette technologie est indissociable à mon sens de la connaissance des milieux dans lesquels opèrent les techniques étudiées, ou en d'autres termes d'études en ethnoscience ${ }^{6}$ où les techniques sont liées aux représentations de la nature ou, pour ce qui est des Kanak, de la cosmogo$n^{7}{ }^{7}$. Car, dans les sociétés kanak comme dans de

4. À ce propos, voir aussi ce qu'en dit Jean-Luc Jamard : « une fonction, entre autres, du mythe étiologique consiste à rendre compte du passage de la nature à la culture ou de la naissance d'une culture propre à notre espèce (car dans les cosmovisions, des animaux sont parfois, eux aussi, "cultivés"). En effet, si c'est le cas, alors le don, le vol ou la découverte de techniques devraient constituer un événement clé des histoires expliquant comment certains êtres devinrent pleinement humains - voir évidemment l'occurrence décisive, souvent inaugurale, que constitue en cela l'obtention du feu dans des récits qui sont légion ailleurs ou jadis. " (2004:\$6).

5. Cette organisation sociale kanak est donc très dépendante des spécialités techniques, comme l’a écrit Sigaut (2003: $\$ 10)$ par ailleurs en discutant la formule de Mauss : "Dire qu’une technique ne peut exister que dans une tradition, c'est dire que pour exister, il faut qu'elle ait été apprise (ou inventée) et qu'elle soit pratiquée par quelqu'un, l'un et l'autre n'étant possibles qu'à l'intérieur de groupes sociaux qui ne sont pas n'importe lesquels, et qui ont donc leurs traditions propres. La morphologie des sociétés, c'est-à-dire la façon dont les humains s'agrègent en groupes et en sous-groupes identifiables et structurés, n'est pas seulement affaire de parenté, de pouvoir, de langage, de religion, etc. Elle est aussi affaire d'apprentissage et de pratiques techniques. "

6. Avec Haudricourt (1985) et Barrau (1973, 1975, 1977), nous mettons ethnoscience au singulier car : "Le terme d'ethnoscience est employé pour embrasser l'ensemble des connaissances qu'une population a de son milieu naturel, interne et externe, animé et inanimé. [...] L'ethnoscience d'une société donnée sera donc décrite à un stade déterminé de son histoire et constituera une portion de l'histoire générale des sciences et des techniques, qui a toujours été pour moi le cadre dans lequel s'inséraient les recherches ethnographiques sur les techniques : pratique et théories. Cependant les connaissances qu'une population a de son milieu impliquent une relation particulière de la société à celui-ci, qui fait également l'objet de l'étude ethnoscientifique. [...] l'ethnoscience a également pour objectif de déterminer les influences et les contraintes que le milieu exerce sur la société et inversement les types d'exploitation et les répercussions de son implantation et de son action sur son environnement. Suivant notre maître, Marcel Mauss, la société formant un système total, tout le groupe social s'exprime dans une étude exhaustive, quel que soit l'aspect par lequel on l'aborde, donc aussi bien par les relations avec les autres vivants, plantes et animaux, par exemple, que par les relations interhumaines." (Haudricourt, 1985 : 7). Voir aussi Leblic (2002, 2005).

7. Pour plus de détails sur ces questions, voir la synthèse que j'en ai réalisée dans mon ouvrage d'anthropologie maritime kanak (Leblic, 2008). 
nombreuses sociétés non occidentales, l'opposition nature/culture n'est pas opérante (Barrau, 1983 ; Descola, 2005 ; Leblic, 2005); l'idée de nature est à l'origine d'une conception ou d'une organisation du monde particulière. Ainsi, pour les Kanak paicî, la nature c'est le pays (näpô) et les hommes et êtres surnaturels qui le peuplent. Selon cette conception kanak de la nature, les esprits, génies et ancêtres, qui interviennent dans toute action humaine comme sur le monde en général, le font aussi par conséquent sur la nature, dans son utilisation comme dans sa gestion. Dans le même ordre d'idées, les interdits jouent un rôle fondamental dans l'insertion du domaine technique dans le social. Ceux-ci ont notamment pour objet de placer les individus qui les respectent dans un état de communion avec les ancêtres qui soutiennent leurs actions et qui ont été sollicités dans les divers rites propitiatoires effectués avant la pratique de toute activité. Dans ce dispositif, l'igname a une place réelle et une symbolique essentielle. C'est André-Georges Haudricourt ${ }^{8}$ (1964) qui le premier souligna la reproduction par bouturage comme $^{9}$ caractéristique de la civilisation de l'igname, mettant ainsi en parallèle les clones et les clans ${ }^{10}$, car :

« [le] clan est pareil à l'igname. Il est le corps social qui, d'époque en époque, se survit à lui-même par bouturage » (Kasarhérou, 1990 : 50)

Et en matière d'interactions entre cosmogonie, croyances, structure sociale et faits techniques, le texte de Maranda et Revolon nous donne un parallèle intéressant concernant les îles Salomon. Comment comprendre l'efficacité de telle technique si l'on ne prend pas en compte l'intervention des non-humains ${ }^{11}$ et de tous les éléments de la surnature pour la réussite de l'opération ${ }^{12}$ ? Il s'agit de quelque chose de fondamental en pays kanak. Aucune pêche par exemple ne peut avoir lieu sans la réalisation au préalable d'un geste, d'une " coutume " ou d'un " tabac " comme on dit, adressé aux esprits ancestraux garants de la réussite de l'activité (Leblic, 1993 : 151, 2000, 2008).

Faut-il rappeler que, dès 1936, Mauss liait les actes techniques, les actes physiques et les actes magico-religieux $^{13}$ qui "sont confondus pour l'agent " (1966: 371) ? Cette question, qui n'a rien d'évident encore actuellement pour nombre

8. En son temps, Maurice Leenhardt (1946: 192-193) avait déjà noté l'importance de la "valeur sociale et religieuse " de la cordyline en pays kanak, qui « dans sa durée, vivace, assure l'efficience permanente d'un sacrifice », sans pour autant à mon sens aller aussi loin qu'Haudricourt dans son article sur les ignames et les clans.

9. "La culture des tubercules est une culture de clone : à chaque saison de culture les mêmes individus sont replantés pour être récoltés à la suivante. Le mot clone désigne l'ensemble des tubercules provenant, par repiquages successifs, du même individu" (Haudricourt, 1964 : 95).

10. "De même que l'origine d'un clone remonte à une plante 'inculte' trouvée en brousse, de même l'origine d'un clan remonte à un 'inculte' arraché de force à la brousse. [...] L'inculte, qui est à l'origine du clan, peut être un étranger " (Haudricourt, $1964: 101-102)$.

11. "La plupart des définitions de la technique font la distinction entre les éléments humains et non humains. Le "non-humain" est généralement associé au contenu matériel, intrinsèque, technique, alors que l'"humain" tend à faire référence aux éléments circonstanciels du contexte (les facteurs sociaux et environnementaux), qui jouent alors le rôle de contraintes dans la transformation de la matière par les moyens techniques. Cette distinction a été contestée par les anthropologues français qui soutiennent que les processus techniques sont enchâssés dans un ensemble plus large de représentations culturelles (Lemonnier, 1993 ; Sigaut 2002), ainsi que par les sociologues qui ont souligné l'importance de la relation analogique entre les deux systèmes, sociaux et techniques, arguant que tous deux avaient la structure des réseaux d'information (Law, 1992). D’autre part, Bruno Latour (1996) et Marilyn Strathern (1991) se sont distingués en envisageant le rôle des matériaux dans la formation de "connexions partielles" entre les deux systèmes. Alors que pour Latour les matériaux sont essentiels dans le maintien des relations entre le social et le technique, Strathern les considère dans leur analogie avec les substances corporelles qui sont déjà conçues comme relationnelles. Ces deux approches - bien que reconnaissant une relation entre le technique et le social - situent le cour de l'action hors de la matière. " (Küchler et Were, 2009 : 191-192). Cette non prise en compte des esprits dans les chaînes opératoires dans les années 1980-1990 a été notée à juste titre par Ludovic Coupaye (2011: 193). Hélène Balfet, quant à elle, avait parlé de " moments ou points critiques" au sein de la chaîne opératoire, dans lequels elle intégrait les procédures magico-religieuses tout en précisant qu'effectivement, à l'époque (livre paru en 1991 mais fruit d'une réflexion des années précédentes), on avait encore du mal à intégrer ce type d'actions ds l'acte technique. Je remercie Hélène Guiot d'voir attiré mon attention sur ce dernier point.

12. En ce sens, je me situe dans la lignée, comme le rappelle Georges Guille-Escuret, d'André-Georges Haudricourt, Jacques Barrau et Maurice Godelier pour qui «les relations entre êtres humains sont indissociables des relations que ceux-ci entretiennent avec la totalité des composantes de leurs milieux. Il n'y a pas de typologie a priori, ni, surtout, de hiérarchie entre les liens homme/homme, homme/végétal, végétal/animal, etc. » (Guille-Escuret, 2003 : \$99) ; et Barrau de noter : "C'est à ces Mélanésiens que je dois surtout d'avoir compris combien la relation des hommes à la nature était indissociable de la relation des hommes entre eux " $(1983: 368)$. C'est en ce sens que j'avais écrit à l'époque que : "Les sociétés kanak peuvent être considérées comme un bon exemple de ce que Pierre Gourou (1948, 1965-1966) a appelé la "civilisation du végétal" et de ce que André-Georges Haudricourt nomme avec plus de précisions la "civilisation de l'igname". L'étude de la manière dont "les types de comportement associés à des modes d'utilisation du milieu naturel ont affecté ou affectent d'autres aspects de la culture" est l'un des objectifs des recherches ethnobotaniques et ethnozoologiques (Barrau, 1975 : 41). ( (Leblic, $2002: 116-117)$.

13. Comme le rappelle Sigaut (2003: 9) : «Dès 1903, Marcel Mauss, s’interrogeant sur les rites magiques, les définissait comme des "actes éminemment efficaces", c’est-à-dire "capables de produire autre chose que des conventions ; [...] ils sont créateurs; ils font". Mais, ajoutait-il, "les techniques elles aussi sont créatrices. Les gestes qu'elles comportent sont également réputés efficaces. À ce point de vue, la plus grande partie de l'humanité peine à les distinguer des rites”. " 
de nos collègues, se trouve même au centre des problématiques les plus actuelles de l'étude des techniques (voir Lemonnier, 2012) : en l'occurrence, explorer les liens étroits entre activité rituelle et activité technique, alors même que l'opposition entre rituel et technique définit traditionnellement le domaine rituel (Lemonnier, 2004). Avec Mauss, rappelons qu'un acte technique est un " acte traditionnel efficace " (1966 : 371):

"J'appelle technique un acte traditionnel efficace (et vous voyez qu'en ceci il n'est pas différent de l'acte magique, religieux, symbolique). Il faut qu'il soit traditionnel et efficace. Il n'y a pas de technique et pas de transmission, s'il n'y a pas de tradition. C'est en quoi l'homme se distingue avant tout des animaux : par la transmission de ses techniques et très probablement par leur transmission orale. » (Mauss, 1966 : 371-372)

Cet auteur divisa les actes techniques en techniques à instrument et techniques du corps. Comme le rappelle Sigaut $(2003,2010)$, dans sa discussion de la formule maussienne, l'acte traditionnel efficace :

" peut être traité comme une formule dans laquelle l'action, la tradition et l'efficacité (à production d'effets physiques) sont les composantes de la technique. [...] le modèle maussien de la technique est présent partout, et [...] sa validité est donc universelle. » $(2003: 168)^{14}$

Mais une autre façon de comprendre la formule de Mauss est, comme le fait Nathan Schlanger (voir édition critique de Mauss, Schlanger, 2012), de la rapprocher du célèbre "fait social total» en parlant de "fait technique total " (1991 : 114). En présentant "la technologie de Mauss telle qu['il] la comprend» (1991: \$3), Schlanger ${ }^{15}$ insiste sur « le thème de la pratique raisonnée, constitutive, en perpétuel devenir" qui traverse " toute l'œuvre de Mauss » :

"On verra que pour Mauss, la technique est fondamentalement sociale, puisqu'elle est une des manifestations du social, un des phénomènes qui le caractérisent. Mais, plus qu'un accompagnement, la technique est aussi un élément fondateur de la société, quelque chose qui la constitue et la conditionne, qui la répète et la façonne. Dans cette acception, la technique va devenir pour Mauss le nom métonymique de la société. »(Schlanger, 1991 : \$4)

Et l'auteur continue son développement sur l'importance sociale de la technologie :
"Mauss rejoint aussi la technologie à un niveau plus profond [...] le fait technique devient faire technique, puis encore faire social. La technique se révèle alors être une de ces pratiques "insidieuses", perpétuellement et profondément impliquées dans le devenir de la société, dans la constitution de sa forme et de sa raison.» (1991 : \$32)

\section{pour conclure :}

"C'est la société qui n'est rien si elle n'est relation, mouvement, devenir, car elle est elle-même une chaîne opératoire, une série d'actes et de représentations montés et remontés collectivement, un savoir tacite qui devient nécessaire, une raison pratique habituelle, perpétuellement efficace puisqu'elle constitue de nouveaux rapports en se pratiquant. » (1991: 62)

Autres matières à réflexion au cour de l'immatériel dans le matériel : les questions de patrimoine autochtone ${ }^{16}$ et la réappropriation d'un patrimoine matériel dispersé. Depuis l'an passé, nous avons consacré plusieurs articles à la question de la restitution des têtes tatouées momifiées māori (voir le JSO I 34 avec les contributions de Gagné, de Boulay, de Peltier et de Mélandri ; et ce volume dans lequel Natacha Gagné revient sur une cérémonie de restitution, à Montréal cette fois-ci, et qui contient aussi la retranscription partielle de la table-ronde qui s'est tenue en mai dernier au festival de cinéma Rochefort Pacifique sur le thème de la "Restitution des restes humains». Ces deux textes sont complétés par l'article en anthropologie juridique de Guillaume Fontanieu qui présente sous un nouvel angle le problème des restitutions, comme nous allons le voir ci-dessous. Ces articles nous permettent d'aborder tant les questions de droit sur les collections - propriété, appropriation, restitution... - des restes humains et d'autres objets des collections muséographiques, que les problèmes de restitution, cette dernière revendication pouvant être vue par certains comme une des formes modernes d'action politique, un juste retour de la colonisation. Ainsi, l'article de Wonu Veys intitulé « Préserver pour la postérité : des anciens de l'ethnie bundjalung au musée national d'ethnologie des Pays-Bas " aborde cette question de restitution plus politique. Ces thématiques amènent une réflexion sur l'avenir (le non-avenir) des musées ethnographiques en tant que tels, sur l'autre en tant que collection / objets menacés (exemples de musées néerlandais). En ce sens, nous espérons avoir dans nos colonnes

14. Sur l'efficacité des techniques de pêche, voir entre autres Leblic (2001).

15. À ce propos, il nous faut remarquer qu'une partie du texte de Schlanger démontre " en quel sens, les "techniques du corps" sont des “techniques" (1991: \$6), ce qui est tout le contraire de la thèse développée par Jean-Michel Le Bot (2009) sur laquelle nous ne attarderons pas ici.

16. Ces dernières années, de nombreux articles de notre Journal ont abordé ces questions de patrimoine et de patrimonialisation (par exemple Le Roux dans le volume 134 ou Faurie dans le volume 132) 
prochainement des contributions relatant des histoires de musées et/ou de collections, mais aucun article sur ce thème n'a pu être achevé pour le présent numéro.

Qu'en est-il aujourd'hui de cette réappropriation, à l'époque où l'unEsCo inscrit au patrimoine mondial de l'humanité de nombreux sites océaniens? Au 10 juin 2013, 962 biens sont inscrits sur cette liste de l'unESCO, dont 745 biens culturels, 188 sites naturels et 29 mixtes avec respectivement, pour ce qui concerne l'Océanie qui nous intéresse ici, 6 biens culturels dont seulement 2 datant d'avant la colonisation européenne (Roi Mata et Rapa Nui), 20 sites naturels et 5 mixtes ( $c f$. le tableau 1).

Un autre point que nous aurions aimé développer dans ce dossier est le dialogue avec la mémoire orale afin de redonner chair à des objets dont l'usage a pu être perdu au fil du temps.
Avec Gilles Bartholeyns, Nicolas Govoroff et Frédéric Joulian, nous rappellerons «l'importance de l'analyse des discours en regard des objets, gestes, savoir-faire et de leur transmission " (2010 : 7). Cette question d'importance n'est pas abordée spécifiquement dans ce volume, pas plus que la mise en lumière des objets techniques du quotidien qui ont disparu des présentations muséographiques actuelles; nous les mentionnaons pour mémoire.

Si aucun texte n'offre l'opportunité d'aborder les objets révélateurs des perceptions occidentales sur le monde kanak depuis les premiers "découvreurs" et le début de la colonisation (des objets de contact), l'article de Roberta Colombo-Dougoud sur les bambous gravés permet d'aborder le thème inverse, la perception par les Kanak de l'univers européen qui est venu bouleverser leur quotidien. En ce sens, les bambous

\begin{tabular}{|c|c|c|c|}
\hline Pays & Sites culturels & Sites naturels & Sites mixtes \\
\hline \multirow{11}{*}{ Australie } & Opéra de Sydney & Baie Shark & Parc national de Kakadu \\
\hline & Sites de bagnes australiens & Côte de Ningaloo & Parc national Uluru-Kata Tjuta \\
\hline & $\begin{array}{l}\text { Palais royal des expositions } \\
\text { et jardins Carlton }\end{array}$ & Parc national de Pumululu & Région des lacs Willandra \\
\hline & & $\begin{array}{l}\text { Site fossilifère des mammiferes } \\
\text { d'Australie (Riversleigh/Naracoorte) }\end{array}$ & $\begin{array}{l}\text { Zone de nature sauvage de } \\
\text { Tasmanie }\end{array}$ \\
\hline & & Tropiques humides de Queensland & \\
\hline & & La Grande Barrière & \\
\hline & & Île Fraser & \\
\hline & & $\begin{array}{l}\text { Forêts humides Gondwana de } \\
\text { l'Australie }\end{array}$ & \\
\hline & & Îles Lord Howe & \\
\hline & & Région des montagnes bleues & \\
\hline & & Îles Heard et McDonald & \\
\hline France & & $\begin{array}{l}\text { Lagons de Nouvelle-Calédonie: } \\
\text { diversité récifale et écosystèmes } \\
\text { associés }\end{array}$ & \\
\hline Vanuatu & Domaine du chef Roi Mata & & \\
\hline \multirow{3}{*}{$\begin{array}{l}\text { Nouvelle- } \\
\text { Zélande }\end{array}$} & & $\begin{array}{l}\text { Te Wahipounamu - zone sud-ouest } \\
\text { de la NZ }\end{array}$ & \multirow{3}{*}{ Parc national de Tongariro } \\
\hline & & Île sub-antarctique de la NZ & \\
\hline & & Île Macquarie & \\
\hline $\begin{array}{l}\text { Royaume- } \\
\text { Uni }\end{array}$ & & Île d'Henderson, archipel de Pitcairn & \\
\hline Kiribati & & Aire protégée des îles Phoenix & \\
\hline Salomon & & Rennell Est & \\
\hline \multirow[b]{2}{*}{ USA } & & Parc national des volcans d'Hawaii & \\
\hline & & $\begin{array}{l}\text { Papahānaumokuākea (vaste groupe } \\
\text { linéaire et isolé de petites îles et atolls } \\
\text { à faible altitude à près de } 250 \mathrm{~km} \\
\text { au nord-ouest du principal archipel } \\
\text { hawaiien) }\end{array}$ & \\
\hline Marshall & $\begin{array}{l}\text { Site d'essais nucléaires de } \\
\text { l'atoll de Bikini }\end{array}$ & & \\
\hline Chili & Parc national de Rapa Nui & & \\
\hline Total & 6 & 20 & 5 \\
\hline
\end{tabular}

TABLEAU 1. - Les sites océaniens inscrits au patrimoine mondial de l'UNESCO (http://whc.unesco.org/fr/list/) 
gravés sont des objets qui ont une signification sociale et/ou historique, racontant certaines parties de l'histoire du contact. Dans le même ordre d'idées, le texte de Dominique Barbe sur l'art sacré catholique kanak nous montre comment les Kanak ont su récupérer et adapter les symboles religieux catholiques.

Une réflexion sur l'articulation entre domaines matériel et immatériel, et entre humain et non-humain, entre rituels et environnement se trouve dans l'article de Pierre Maranda et Sandra Revolon qui traite de la cosmologie dans l'objet à propos de la part "d'immatériel » dans l'objet de culture dite "matérielle» qu'est la Barque blanche de Malaita aux îles Salomon, pirogue rituelle dont la construction par des non-chrétiens fut observée en 1968 par Maranda ; en parallèle, Revolon présente une dynamique immatérielle comparable en milieu chrétien parmi les Owa d'Aorigi. Ce texte de technologie rituelle permet de saisir tant les modes de pensée et de vie de ces deux sociétés des Salomon que les processus techniques de fabrication de pirogues cérémonielles. De même, l'article de Serge Tcherkézoff montre comment les nattes fines de Samoa ont une valeur immatérielle et sont comme une monnaie au sens maussien du terme.

L'article de Christian Coiffier sur les fougères ornant les manches des casse-têtes kanak nous permet d'aborder les pratiques et les réflexions autour des patrimoines matériels et immatériels en révélant le lien entre objets et pratiques ou le rôle occulte de la fougère qui n'était sans doute pas là uniquement pour décorer le casse-tête. Il montre aussi que les objets mettent en lumière l'articulation entre culture matérielle, pratiques rituelles et environnement, à travers la présentation des processus techniques ou des savoirs locaux et rituels, sans oublier les processus de transmission, tout thèmes au cœur des patrimoines matériels et immatériels des sociétés kanak et océaniennes.

\section{Présentation des articles}

Les trois premiers articles sont centrés sur la valeur idéelle ou immatérielle de certains objets techniques. Pour ouvrir notre dossier, Pierre Lemonnier revient sur les notions d'immatériel et de matériel (pp. 13-24) et «sur la faculté des objets et des techniques à combiner pensées et actions ", en partant des notions d'idéel et de matériel chères à Maurice Godelier (1984).

«Envisager la part d'immatériel, d'imaginaire et de pensée qui se niche dans un objet évoque immédiatement deux thèmes de recherche anthropologique : les réflexions de Maurice Godelier (1984) sur la prise en compte de "l'idéel" dans la description et l'analyse des forces productives, bien sûr, mais aussi la proposition inverse, relative à la part jouée par certains éléments d'une culture matérielle, objet ou simple technique du corps, dans l'idéel ; en l'occurrence, dans l'expérience et la reproduction d'un imaginaire partagé. En anthropologie, l'une et l'autre manières de rapprocher le monde des objets et des actions matérielles de celui des idées sont historiquement liées, mais la seconde s'interroger sur le rôle des objets dans la constitution du système de représentations temporairement propre à une société - ouvre des perspectives nouvelles. En vérité, c'est, depuis peu, l'une des façons d'intégrer définitivement l'étude des objets et des techniques dans l'anthropologie la plus générale en apportant à celle-ci des résultats qu'il n'est pas possible d'obtenir autrement. C'est cette transformation radicale de l'anthropologie des objets dans les quarante dernières années que je me propose de résumer en ayant à l'esprit les rapports entre matériel et immatériel. » (p. 15)

En se fondant sur l'histoire de la technologie culturelle et de l'anthropologie économique marxiste, des faits ethnologiques et des objets produits par les sociétés traditionnelles à la fois idéels et matériels, l'auteur rappelle que certains sont de véritables expressions physiques de ce que Mauss appelle les phénomènes sociaux totaux dans lesquels s'exprime l'ensemble des institutions et croyances. Ces objets particuliers, par le lien interactif et réciproque entre objet matériel et conception idéelle, permettent de percevoir par une communication non verbale cette totalité sociale liée à une conception particulière de l'univers et de la place de l'homme dans celui-ci.

Ce lien sera fait tout particulièrement dans l'article suivant de Pierre Maranda et Sandra Revolon, "La cosmologie dans l'objet... " (pp. 27-42), où les auteurs montrent l'importance symbolique, cognitive et sociale des objets matériels et immatériels des barques de pêche décorées et leurs liens avec les mythes, la littérature orale et les traditions locales. La série photographique appelle une chaîne opératoire qui ne se trouve malheureusement pas dans l'article par manque de place. Cet article montre comment deux sociétés des îles Salomon relient les techniques à des mondes immatériels omniprésents, que ce soient le système magico-religieux traditionnel (Lau) ou son insertion dans la religion catholique (Owa). Les auteurs avancent ici des critiques à l'état de questionnement sur les théories de l'" aesthetic captivation " et des "technologies of enchantment", de la mystification, etc.

Puis, Serge Tcherkézoff (pp. 43-62) nous présente la valeur sociale d'un objet presque immatériel, les nattes fines de Samoa, qu'il assimile à une monnaie au sens maussien, car leur valeur immatérielle réside dans la généalogie tressée. Les Samoans tirent une "force" (mana) remarquable de ces nattes fines : elles permettent la réalisation des funérailles d'un mort au loin, 
elles peuvent compenser une vie perdue à la suite d'un meurtre et elles englobent tout don initial en étant le contre-don par excellence. Au final, l'auteur nous montre comment elles peuvent être la manifestation visuelle des pouvoirs de vie, au sens hocartien, que chaque société doit pouvoir manipuler pour s'autoriser à croire en sa pérennité.

Les quatre articles qui suivent présentent une autre facette de notre dossier, en abordant la question des restitutions des restes humains, d'abord au musée de Leyde aux Pays-Bas avec le texte de Wonu Veys (pp. 63-76). L'auteur y montre comment des objets aborigènes, devenus obsolètes aujourd'hui, ont le pouvoir, par leur présence et leur part d'immatériel, de provoquer la mémoire. Elle en conclut la nécessité d'engager une réflexion plus ample sur le rôle médiateur présent et futur des musées ethnographiques. Natacha Gagné (pp. 77-88) continue la série d'articles commencée dans nos pages sur les cérémonies de restitution de toi moko... aux Māori de Nouvelle-Zélande, d'abord celle du musée du quai Branly (voir JSO 134) et, cette fois-ci celle du musée des Beaux-Arts de Montréal. Dans ce texte, elle s'interroge notamment sur les différences entre les deux cérémonies de restitution qui sont très liées aux contextes nationaux français et canadien. Suit la retranscription du débat sur les restitutions qui eut lieu au festival de Rochefort (pp. 89-102) et qui amène des points de vue intéressants sur cette question des restitutions en général. Enfin, Guillaume Fontanieu revient sur « La restitution des mémoires, une expérience humaine, une aventure juridique " (pp. 103-118) de son point de vue d'anthropologue juridique (qui est plus juridique qu'anthropologique) et sur la question de la restitution du crâne du chef kanak Ataï, un des meneurs de l'insurrection de 1878 devenu depuis un mythe en milieu kanak, comme le montrent les deux textes publiés hors dossier d'Alain Saussol et d'Hamid Mokkadem. Fontanieu, au-delà de la question de la restitution particulière du crâne d'Ataï, revient sur la conception du droit français sur la personne après la mort, considérée comme une chose sacrée, en la comparant avec la vision des Kanak sur le corps afin de faire émerger l'hypothèse d'une nouvelle conceptualisation autour de la notion de non-personne. Le texte de Saussol (pp. 169-180) revient en effet sur l'alliance des auxiliaires de Canala dans la répression de l'insurrection de 1878 en tentant de rétablir la réalité des faits et en insistant sur le fait que les Canala ne furent ni les "alliés " ni les « traîtres " que l'on a présentés ainsi pendant longtemps et trop rapidement. Quant à Mokkadem (pp. 181-193), il revient sur la figure d'Ataï à propos de la trajectoire politique d'Eloi Machoro.

Les quatre derniers textes du dossier s'attachent plus spécifiquement au patrimoine kanak. Ainsi, Roberta Colombo-Dougoud, dans «Les bam- bous gravés, représentations de soi et de l'autre " (pp. 119-132), revient sur le parcours d'une exposition, de Genève et Nouméa, pour analyser le concept de patrimoine dispersé et d'objets ambassadeurs de la culture kanak. Puis, Christian Coiffier (pp. 133-148) présente des casse-têtes kanak et leurs ornements végétaux, longtemps passés sous silence. Son article questionne la raison de l'utilisation de telles fougères plutôt que telles autres et s'interroge sur leurs significations en comparant cet usage particulier à la présence de ces mêmes végétaux sur d'autres objets ou dans certains rituels. Enfin, Emmanuel Kasarhérou (pp. 149-152) propose une "Petite histoire d'une acquisition pour le musée de NouvelleCalédonie » à propos de l'achat d'une statuette, très probablement liée au chef Goodu. Dans un autre contexte, Dominique Barbe présente "L'art sacré catholique kanak ou l'immatériel préféré » ou comment l'art kanak a été réutilisé par la religion catholique et comment cette dernière a également pesé sur certaines réalisations kanak, signes d'une certaine superposition des croyances kanak et de la religion chrétienne.

Suivent les rubriques habituelles du Journal, deux autres articles hors dossier, celui d'Émilie Dotte et al. : "Archéogéographie et processus de territorialisation en Nouvelle-Calédonie " (pp. 195-208) et celui de Guillaume Molle et Eric Conte: "Approche techno-typologique des peignes à tatouer... » (pp. 209-226). Deux textes figurent dans les Miscellanées, le premier de Gilles Bounoure, "Arts océaniens, anciens et nouveaux paramètres» (pp. 227-232), et le second, par Christian Coiffier, sur "les rêves océaniens de Jacques et Betty Villeminot" (pp. 2313-238).

Enfin, pour terminer ce volume, outre les comptes rendus d'ouvrage, nous présentons malheureusement deux nécrologies In memoriam en l'honneur de collègues décédés, Michel Panoff (par Georges Guille-Escuret) et Darrell Tryon (par Alexandre François et Serge Tcherkézoff). Le compte rendu de notre assemblée générale 2013 qui a vu l'élection du nouveau conseil d'administration de la société clôt définitivement l'ensemble. Et nous terminons ce volume par la publication d'une de nos conférences, celle donnée par Sandhya Patel le 21 mars 2013, «Les rencontres de James Cook et Joseph Banks ", qu'elle a repris pour nous proposer cette note de recherche.

\section{BIBLIOGRAPHIE}

BaLfet Hélène, 1991. Incident et maîtrise technique dans les chaînes opératoires, in H. Balfet et al., Observer l'action technique : 
des chaînes opératoires, pour quoi faire?, Paris, Éditions du CNRS, pp. 179-187.

Balfet Hélène et al., 1991. Observer l'action technique: des chaînes opératoires, pour quoi faire?, Paris, Éditions du CNRS.

Bartholeyns Gil, Nicolas Govoroff et Frédéric Joulian, 2010. Introduction, Techniques \& Culture 54-55: Cultures matérielles, pp. 1-13 (http://tc.revues.org/pdf4972).

Barrau Jacques, 1973. Plantes et comportements des hommes qui les cultivent. L'œuvre ethnobiologique d'André Haudricourt, La Pensée 171 : Spécial Ethnologie, pp. 37-46.

—, 1975. Chapitre 11. Écologie, Eléments d'ethnologie. 2. Six approches, in Robert Cresswell et al., Paris, Armand Colin, coll. U, pp. 7-43.

-, 1977. Histoire naturelle et anthropologie, L'Espace géographique 3, pp. 203-209.

-, 1983. LES Hommes et leurs aliments, Esquisse d'une histoire écologique et ethnologique de l'alimentation humaine, Paris, Messidor/ Temps Actuels.

Chamoux Marie-Noëlle, 1983. La division des savoir-faire textiles des Indiens et Métis dans la Sierra Puebla (Mexique), Techniques \& Culture 2, pp. 99-124.

Coupaye Ludovic, 2009. Décrire des objets hybrides. Les grandes ignames décorées du village de Nyamikum, province de l'East Sepik, Papouasie-Nouvelle-Guinée, Techniques \& Culture 52-53: Technologies, pp. 50-67 (http://tc.revues.org/4730).

—, 2011. Anthropologie de l'objet, anthropologie par l'objet : profils théorique et profils matériels des ignames Décorées des Nyamikum Abelam (Papouasie-Nouvelle-Guinée), in F. Wateau, C. Perlès, C. Soulier et P. Soulier (éds), Profils d'Objets : Approches d'Anthropologues et d'Archéologues, Paris, De Boccard, pp. 187-199.

—, 2012. Des images, des nouds et des toiles. Pierres sacrées et ignames à Nyamikum (district de Maprik, East Sepik Province, Papouasie-Nouvelle-Guinée), Techniques \& Culture 58: Objets irremplaçables, pp. 142159.

Coupaye Ludovic et Laurence Douny, 2009. Dans la trajectoire des choses. Comparaison des approches francophones et anglophones contemporaines en anthropologie des techniques, Techniques \& Culture 52-53 : Technologies, pp. 12-39 (http://tc.revues.org/4956).

Cresswell Robert, 1994. La nature cyclique des relations entre le technique et le social.
Approche technologique de la chaîne opératoire, in Bruno Latour et Pierre Lemonnier (éds), De la préhistoire aux missiles balistiques. Lintelligence sociale des techniques, Paris, La Découverte, pp. 275-289.

DAMON Frederick, 2008. On the Ideas of a Boat: From Forest Patches To Cybernetic Structures, in C. Sather and T. Kaartnen (eds), Beyond the horizon: essays on myth, history, travel and society, Helsinki, Finnish Literature Society, Studia Fennica Anthropologica 2, pp. 123-144.

Descola Philippe, 2005. Par-delà nature et culture, Paris, Gallimard, Bibliothèque des sciences humaines.

FAURIE Mathias, 2011. Impacts et limites de la patrimonialisation à Ouvéa (Nouvelle-Calédonie), Journal de la Société des Océanistes 132, pp. 109-122.

Guille-Escuret Georges, 2003. Efficacité technique, efficacité sociale. Le technique est-il dans le social ou face à lui ?, Techniques \& culture 40: Efficacité technique, efficacité sociale, np (http://tc.revues.org.gate3.inist. fr/1414).

Haudricourt André-Georges, 1964. Nature et culture dans la civilisation de l'igname: l'origine des clones et des clans, L'Homme 4, pp. 93-104.

—, 1985. Avant-propos : À propos de l'ethnoscience, in Serge Bahuchet, Les Pygmées Aka et la forêt centrafricaine. Ethnologie écologique, Paris, SELAF et CNRS, coll. Ethnoscience 1, p. 7.

—, 1987. La Technologie, science humaine. Recherche d'histoire et d'ethnologie des techniques, Paris, Éditions de la Maison des sciences de l'homme.

JAMARD Jean-Luc, 2004. Avant-propos. Mythes et techniques, ou l'origine des manières de faire, Techniques \& Culture 43-44: Mythes. L'origine des manières de faire, np (http:// tc.revues.org/975).

Juillerat Bernard, 2004. À l'origine des techniques, l'interdit? Escales océaniennes, Techniques \& Culture 43-44: Mythes. L'origine des manières de faire, np (http://tc.revues. org/976).

KASARHÉROU Emmanuel, 1990. Les saisons et les jardins, De jade et de nacre. Patrimoine artistique kanak, Paris, Réunion des musées nationaux, pp. 50-67.

KüChler Susanne et Graeme Were, 2009. Empathie avec la matière. Comment repenser 
la nature de l'action technique, Techniques \& Culture 52-53 : Technologies, pp. 190-211 (http://tc.revues.org/4942).

Latour Bruno, 1996. Lettre à mon ami Pierre sur l'anthropologie symétrique, Ethnologie française 26, 1, pp. 32-36.

LAw John, 1992. Notes on the theory of the Actor-network: ordering, strategy, ans heterogeneity, Systems Practice 5, 4, pp. 379-393.

Leblic Isabelle, 1988. Évolution des techniques de pêche en Nouvelle-Calédonie, Techniques \& culture 12: Symboles et procès techniques, pp. 81-119.

_, 1999. Marmites rituelles et autochtonie à Ponérihouen (Vallées de Göièta-Näbai, Nouvelle-Calédonie), Techniques \& Culture 33 : Entre histoire et tradition, pp. 53-87.

-, 2000. Une pirogue pontée à l'île des Pins, Techniques \& Culture 35-36 : Traversées. Construction navale, expressions symboliques Asie-Pacifique, pp. 301-326.

—, 2001. Efficacité technique et rituelle face aux changements techniques et sociaux : le cas des pêcheurs kanak de Nouvelle-Calédonie, in G. Danic, A. Geistdoerfer, G. Le Bouëdec et F. Théret (éds), Mutations techniques des pêches maritimes: agir ou subir ?, Brest, IFREMER, actes de colloques 30, pp. 135-164.

-, 2002. Ignames, interdits et ancêtres en Nouvelle-Calédonie, Journal de la Société des Océanistes 114-115: En hommage à Jacques Barrau, pp. 115-127.

—, 2005. Pays, "surnature " et sites "sacrés" paicî à Ponérihouen (Nouvelle-Calédonie) (com. au coll. unEsCo 1998), Journal de la Société des Océanistes 120-121 : Ethnoécologie en Océanie, pp. 95-111.

-, 2008. Vivre de la mer, vivre avec la terre... en pays kanak. Savoirs et techniques des pêcheurs kanak du sud de la Nouvelle-Calédonie, $\mathrm{Pa}-$ ris, Société des Océanistes, Travaux \& documents océanistes 1 .

Le Bot Jean-Michel, 2009 (4/07). À propos des "techniques " du corps : retour sur larticle de Mauss (http://halshs.archives-ouvertes.fr/ halshs-00401717/).

Lemonnier Pierre, 1993. Technological choices. Transformation in material cultures since the Neolithic, Londres, Routledge.

—, 2004. Mythiques chaînes opératoires, Techniques \& Culture 43-44: Mythes. L'origine des manières de faire, np (http://tc.revues. org/1054).
—, 2011a. Fallait-il en passer par là ? Du grand silence des forces productives en anthropologie marxiste aux joies de la technologie culturelle rénovée, in N. Barbe et J.-F. Bert (eds), Penser le concret. André Leroi-Gourhan, André Georges Haudricourt, Charles Parain, Paris, Créaphis Éditions, pp. 81-88.

—, 2011b. Faire penser : une dimension maltraitée des objets, Le Genre humain 50 : L'archéologie comme discipline? Du colloque à la publication, édité par Ph. Boissinot, pp. 71-86.

-, 2012. Mundane Objects. Materiality and Nonverbal Communication, Walnut Creek (California), Left Coast Press.

Leroi-Gourhan André, 1952. Sur la position scientifique de l'ethnologie, La revue philosophique, Paris, PUf, pp. 506-518.

Le Roux Géraldine, 2012. Regards d'artistes sur les processus de patrimonialisation et de commercialisation de la culture aborigène, Journal de la Société des Océanistes 134, pp. 85-94.

Mauss Marcel, 1966. Les techniques du corps, in Sociologie et anthropologie, Paris, PUF, pp.

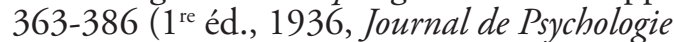
XXXII, 3-4, pp. 271-293)

Schlanger Nathan, 1991. Le fait technique total. La raison pratique et les raisons de la pratique dans l'œuvre de Marcel Mauss, Terrain 16: Savoir-faire, pp. 114-130 (http://terrain. revues.org/3003).

Schlanger Nathan (éd.), 2012. Techniques, technologies et civilisation. Marcel Mauss, édition et présentation de Nathan Schlanger, Paris, puf, Édition critique de Marcel Mauss 8.

Sigaut François, 2002. Technology, in tim ingold (ed.), Companion Encyclopedia of Anthropology, Londres, Routledge, pp. 420-459.

_, 2003. La formule de Mauss, Techniques \& culture 40 : Efficacité technique, efficacité sociale, pp. 153-168 (http://tc.revues.org. gate3.inist.fr/1538).

—, 2010. Retour sur la formule de Mauss, Techniques \& culture 54-55: Efficacité technique, efficacité sociale, pp. 354-356.

Strathern Marilyn, 1991. Partial connections, Savage, MD, Rowman \& Littlefield.

WARniER Jean-Pierre, 2009. Les technologies du sujet. Une approche ethno-philosophique, Techniques of Culture 52-53: Technologies, pp. 148-167 (http://tc.revues.org/4853). 
